\title{
Memory Effects in Backscattering of Two-Dimensional Electrons in Corrugated Systems
}

\author{
N. M. Sotomayor *, J. F. da Rocha Neto *, and G. M. Gusev ${ }^{\dagger}$ \\ * Universidade Federal do Tocantins, Campus Universitário de Arraias and \\ $\dagger$ Instituto de Física da Universidade de São Paulo, Caixa Postal 66318, Brazil
}

Received on 4 April, 2005.

\begin{abstract}
It is reported a theoretical and numerical study of non-markovian memory effects in backscattering of ballistic electrons constrained to move in a corrugated surface topography. Two approaches to model the electron trajectories are used, better approximation is obtained with the Hamilton-Dirac method for constrained system.
\end{abstract}

Keywords: Backscattering of two-dimensional electrons; Numerical study; Memory effects

\section{INTRODUCTION}

The classical low-field magnetoresistance (MR) in metals and semiconductors has been recently revised, since it was recognized that the conventional Boltzmann-Drude approach fails to describe the electron dynamics in disordered systems [1-3]. According to the Boltzmann-Drude model, magnetoresistance must yield no change, in the presence of short range electrostatic potential, which is in disagreement with the facts observed in experiments [4-6]. In a recent work, we report measurements of large linear negative magnetoresistance (LNMR) in a $G a A s / A l_{x} G a_{1-x} A s$ two-dimensional electron system (2D) with non-planar topography, caused by random distribution of corrugations, generated by a combination of pre-patterning and regrowth processes [7]. The observed LNMR, that is an anomalous behavior, reached up to $20 \%$ of the zero field resistivity $\left(\rho_{x x}(B=0)\right)$, however, in a very small region around $B=0$, the resistivity showed a non-anomalous behavior. Recent theoretical developments for the conductivity of a classical two-dimensional Lorentz gas $[8,9]$ allowed us to attribute this MR to non-Markovian memory effects originated by specific return processes in backscattering of electrons by corrugations and defects. However, a certain difference related to the order of magnitude of the full variation of $\Delta \rho_{x x} / \rho_{x x}(0)=\left[\rho_{x x}(B)-\rho_{x x}(0)\right] / \rho_{x x}(0)$ appeared between the theoretical model and our experimental results. This phenomenon that may be closely related to a specific "corridor effect" for scattering with corrugations and antidots, that should preset greater cross sections for electrons, motivate us to explore further the classical electron dynamics of $2 \mathrm{D}$ electrons constrained to random-shape topographies in order to analyze the influence of memory effects that appear in different scattering processes in these systems.

\section{CONSTRAINED DYNAMICS}

For a proper description of the low field magnetoresistance in our samples it is necessary to simulate numerically the dynamics of two-dimensional electrons constrained to move in a randomly-shaped non-planar topography in the presence of a uniform perpendicular magnetic field. A first approach to describe the classical electron dynamics in corrugated semi- conductor systems was made in reference [10], where the case of random magnetic field was treated departing from the Lagrangian of the system. In the present work we use two different approaches to the study the electron dynamics in constrained electron systems to non-planar topographies. As a first model to describe the magnetoresistance of $2 \mathrm{D}$ electrons constrained to random topographies, we assume that the electron motion, influenced by the perpendicular uniform magnetic field, in the non-planar topography formed by the random distribution of corrugations and defects, can be compared with the simulation of a two-dimensional Lorentz gas composed of electrons and hard scatterers, under the influence of a perpendicular uniform magnetic field. The array of nonoverlapping scatterers is characterized by a sum of gaussian potentials with a random distribution. As we are interested in the classical nature of transport in this system, we used linear response theory [11], for the calculation of magnetoresistance $\rho_{x x}$ through a different numerical approach. We started with the Hamiltonian for the classical electron dynamics of a 2D electron gas under the influence of uniform magnetic field, directed perpendicularly to the plane of the gas (Lorentz gas),

$$
H=\frac{1}{2 m^{*}}(\vec{p}-e \vec{A})^{2}+U_{w}(x, y)+U_{2 D}(x, y),
$$

where the potential vector is written as: $\vec{A}=$ $(-B y / 2, B x / 2,0) . \quad U_{w}(x, y)=U_{0}\left(x^{n}+y^{m}\right)$ is a sum of hard potential walls, along $x$ e $y$ directions, to account for periodic conditions, $U_{0}$ is the amplitude and, $n$ and $m$ are positive integers. The random antidot potential of the antidots $U_{2 D}$, is simulated by the expression:

$$
U_{A D}(x, y)=\sum_{i=1}^{M} U_{0} \exp \left[-\left(\frac{x-x_{0}}{\Gamma_{x}}\right)^{\gamma}\right] * \exp \left[-\left(\frac{y-y_{0}}{\Gamma_{y}}\right)^{\gamma}\right]
$$

where $M$ is the number of antidots, $U_{0}$ is the maximum amplitude of the potential of each scatterer and, the parameter $\Gamma_{x}$ and $\Gamma_{y}$ accounts for the antidot diameter at the Fermi energy, and $\gamma$ allows to vary between soft and hard potential profiles, for our calculations we employed $\gamma \sim 2-6$. We used dimensionless variables in similar way as those explained in reference [7]. Four equation of motion are obtained and they were numerically integrated to obtain the electron trajectories. According to classical linear response theory the conductivity of 
the $2 \mathrm{D}$ electron gas is given by:

$$
\sigma_{i j}=\frac{N_{s} e^{2}}{E_{F}} \int_{0}^{\infty}\left\langle v_{i}(t) v_{j}(t=0)\right\rangle_{\Gamma} e^{-\frac{t}{\tau}} d t
$$

where $N_{s}$ is the electron concentration, $E_{F}$ is the Fermi energy, $\left\langle v_{i}(t) v_{j}(0)>_{\Gamma}\right.$ is the velocity-velocity correlation function double averaged over phase space $\Gamma$, the indices $i$ and $j$ stand for the $x$ and $y$ direction, respectively. The presence of additional scattering is included through the electron mean scattering time $\tau$, where the probability of an electron not suffering a collision within the time interval $[0, t]$ is given by $e^{-t / \tau}$. From the numerical computation of the conductivity tensors we are able to determine the longitudinal $\rho_{x x}$ and transverse $\rho_{x y}$ resistivities. Ensembles of electron trajectories, depart inside a square region of side $a_{l}$, where $a_{l}$ is the antidot lattice period, chosen to be equal to unity. This region contains 500 scatterers, with gaussian potentials, placed according to a uniform distribution. The ratio $a / l$ (where $a$ is the radius of the scatterer at the Fermi energy) was chosen to be 0.001 , and the mean free path $l=(N 2 a)^{-1}$ was $6.3 a_{l} . \quad N$ is the twodimensional concentration of scatterers. For the integration process we generate two hundred trajectories for each particular configuration of scatterers, after this a new configuration is established and the process is repeated up to ten times. The 2D electrons move in a medium composed of an array of nonoverlapping and randomly distributed, hard-scatterers. We integrate, numerically, the motion equations for this Lorentz gas and from this we obtained the relative magnetoresistance (MR), which is shown in Fig. 1.

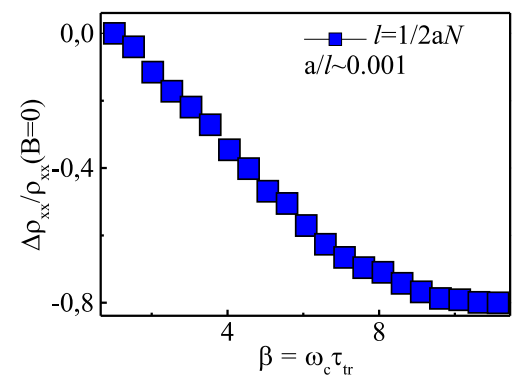

FIG. 1. Relative magnetoresistance for a two-dimensional Lorentz gas obtained by numerical integration.

God agreement with the LNMR experimental results was found. However, a certain difference between this theoretical model and our experimental results is related to the order of magnitude of the full variation of the relative magnetoresistance. While in our experiment the LNMR reaches up to $30 \%$ of its zero value our numerical results show an almost $80 \%$ decrease, that is a better approximation than the results obtained in reference [9]. A more exact approach to describe the electron dynamics of a single electron constrained to move in corrugated surfaces is obtained through the application of the Hamilton-Dirac method for systems including second class constraints [12]. According to this model, the extended Hamiltonian of the system is given by

$$
H_{e}(\vec{p}, \vec{r})=H_{c}-\Phi_{a} \mathbf{C}_{a b}^{-1}\left\{\Phi_{b}, H_{c}\right\}
$$

where $\vec{r}=(x, y, z), \vec{p}=\left(p_{x}, p_{y}, p_{z}\right)$ are the position and momentum vectors respectively. The indexes $a$ and $b$ assume the values 1,2. $\Phi_{1}=\phi=z-\cos ^{\beta}(x)=0$ and $\Phi_{2}=\frac{1}{m} \vec{P} \cdot \vec{\nabla} \Phi_{1}=$ 0 are second class constraints with $\vec{P}=\vec{p}-e \vec{A}$ being $A=$ $\frac{B}{2}(-y, x, 0)$ the potential vector. The matrix $\mathbf{C}_{a b}^{-1}$ is the inverse matrix obtained out of the Poisson brackets $\left\{\Phi_{a}, \Phi_{b}\right\}$. The canonical Hamiltonian $H_{c}$ appearing in 4 is give by

$$
H_{c}(\vec{p}, \vec{r})=\frac{1}{2 m}(\vec{p}-e \vec{A})^{2}-\lambda \phi
$$

where $\lambda$ is a Lagrange multiplier given by

$$
\lambda=\frac{1}{m\left(\vec{\nabla} \Phi_{1}\right)^{2}}\left(\vec{P} \cdot \vec{\nabla}\left(\vec{P} \cdot \vec{\nabla} \Phi_{1}\right)+\frac{1}{2} \vec{\nabla} \Phi_{1} \cdot \vec{\nabla} \vec{P}^{2}\right)
$$

The motion equations of the particle are obtained in terms of the Dirac brackets [12] and are given by:

$$
\dot{\vec{r}}=\frac{1}{m}\left(\vec{P}-\frac{1}{\left(\vec{\nabla} \Phi_{1}\right)^{2}} \vec{\nabla} \Phi_{1}\left(\vec{P} . \vec{\nabla} \Phi_{1}\right)\right)
$$

and

$$
\dot{\vec{p}}=\frac{1}{m}\left(\frac{-1}{2} \vec{\nabla} \vec{P}^{2}-m \lambda \vec{\nabla} \Phi_{1}+\frac{1}{2\left(\vec{\nabla} \Phi_{1}\right)^{2}} \vec{\nabla}\left(\vec{P} \cdot \vec{\nabla} \Phi_{1}\right)^{2}\right) .
$$

The numerical integration of this coupled differential equations gives us the trajectories of the electron scattering in constrained non-planar topographies as the one given by $\phi=$ $z-\cos ^{\beta}(x)=0$. Fig. 2 shows a scheme of this surface together with some electron trajectories calculated for different values of the magnetic field. Scattering by periodic and random arrays of antidot can be added, as well as a random shaped surfaces that can be modeled by the addition of gaussian potentials with negative-positive amplitudes and random distribution. Calculation of magnetoresistance for these systems is under development and will not be presented here.

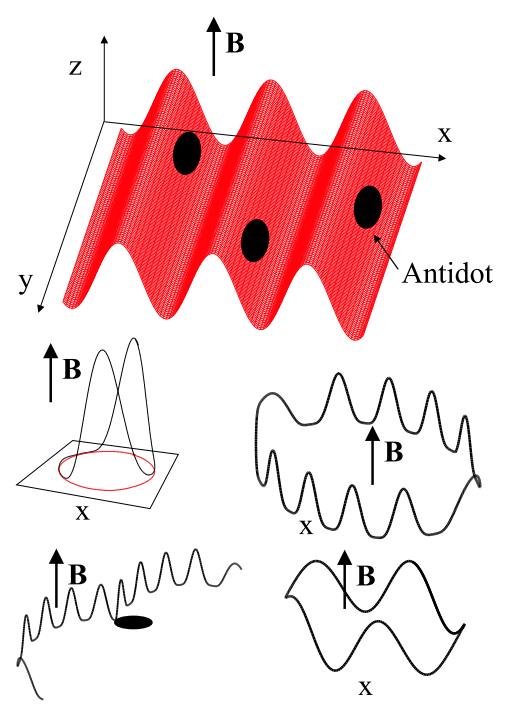

FIG. 2. Scheme of a simple corrugated surface with antidots, and electron trajectories constrained to the non-planar topography. 


\section{CONCLUSION}

In summary, we have modeled the low field electron dynamics of two dimensional electrons constrained to move in non-planar topographies composed of random corrugations and defects, contrary to the prediction of the BoltzmannDrude approach, the MR of one of this disordered system shows a dominant large linear negative decrease. A more exact approach, for the electron dynamics in constrained systems is developed though the Hamilton-Dirac equation.

\section{Acknowledgments}

This work was supported by $\mathrm{CNPq}$ Brazilian Funding Agency and is acknowledged. We would like to thanks, also, to the LCCA of the University of São Paulo by the computational facilities which made possible part of this work.
[1] A. V. Bobylev, Frank A. Maaø, Alex Hansen, and E. H. Hauge, Journal of Statistical Physics. 87, 1205 (1997).

[2] A. D. Mirlin, J. Wilke, F. Evers, D. G. Polyakov, and P. Wölfle, Phys. Rev. Lett. 83, 2801 (1999).

[3] D. G. Polyakov, F. Evers, A. D. Mirlin, and P. Wölfle, Phys Rev. B 64, 205306-1 (2001).

[4] G. M. Gusev, P. Basmaji, Z. D. Kvon, L. V. Litvin, Yu. V. Nastaushev, and A. I. Toropov, Surface Science 305, 443 (1994).

[5] G. M. Gusev, A. A. Quivy, J. R. Leite, A. A. Bykov, N. T. Moshegov, V. M. Kudryashev, A. I. Toropov, and Yu. V. Nastaushev, Semicond. Sci. Technol. 14, 1114 (1999).

[6] A. A. Bykov, G. M. Gusev, J. R. Leite, A. K. Bakarov, A. V. Goran, V. M. Kudryashev, and A. I. Toropov, Phys. Rev. B 65, 035302 (2001)

[7] N. M. Sotomayor, G. M. Gusev, J. R. Leite, A. A. Bykov, A. K. Kalagin, V. M. Kudryashev, and A. I. Toropov, Phys. Rev. B 70,
235326-1 (2004).

[8] A. Dmitriev, M. Dyakonov, and R. Jullien, Phys. Rev. Lett. 89, 266804 (2002)

[9] Vadim V. Cheianov, A. P. Dmitriev, and V. Yu. Kachorovskii, Phys. Rev. B 68, 201304-1 (2003).

[10] M. Trinidade Dos Santos, G. M. Gusev, J. R. Leite, A, A, Bykov, N. T. Moshegov, V. M. Kudryashev, A. I. Toropov, and Yu. V. Nastaushev, Superlattices and Microstructures 25, 167 (1999).

[11] R. Kubo, M. Toda and N. Hashitsime, Statistical Physics II, Springer-Verlag, Berlin (1985).

[12] P. A. M. Dirac, Lectures on Quantum Mechanics, Belfer Graduate School of Science, Yeshiva University (New York, N.Y., 1946). 